\title{
Pilotprojekt „Steirischer Abfallspiegel“
}

\author{
HR DI Dr. Wilhelm Himmel, Amt der Steiermärkischen Landesregierung, FA 19D, Graz
}

Die Fachabteilung 19D - Abfallund Stoffflusswirtschaft hat im Auftrag von Landesrat Johann Seitinger im Sommer 2008 allen steirischen Gemeinden das Angebot gemacht, Kennzahlen über die abfallwirtschaftlichen Dienstleistungen, Kosten, Erlöse und Gebühren in ein elektronisches Datenformular einzutragen und sie eingeladen, an einer landesweiten statistischen Auswertung (Benchmarkstudie "Steirischer Abfallspiegel“) teilzunehmen.

Insgesamt haben 67 steirische Gemeinden und 5 interessierte $\mathrm{Ge}$ meinden aus anderen Bundesländern dieses Angebot angenommen und sich an diesem Projekt beteiligt. Die FA19D bedankt sich auf diesem Weg nochmals sehr herzlich bei allen teilnehmenden Gemeinden, denn es ist nicht selbstverständlich, dass sensible Daten einer Gemeinde - verbunden mit einem erheblichen Eingabeaufwand - im Rahmen eines landesweiten $\mathrm{Pi}$ lotprojektes zur Verfügung gestellt werden. Wir wissen das Vertrauen, welches der FA19D seitens der teilnehmenden Gemeinden entgegengebracht wurde, sehr zu schätzen.

Die Gemeinden haben den wesentlichen Dateninput geliefert und damit den Grundstein für eine erfolgreiche Projektdurchführung geliefert. Im Oktober 2008 wurde den Gemeinden ein umfassender Endbericht übermittelt. Dieser Bericht umfasste 2 Teile, im „allgemeinen“ Berichtsteil wurden überblicksmäßig die Auswertungen für die Sammlung von Restabfall, Sperrmüll, Bioabfall, Altpapier und den Betrieb von Altstoffsammelzentren zusammengefasst, im Abschnitt 2 wurden die „gemeindespezifischen, vertraulichen" Ergebnisse im Sinne eines Ampelsystems mit den steirischen Durchschnittswerten verglichen.

Die allgemeinen Erkenntnisse dieses Projektes wurden am 15. Oktober 2008 in Lannach rd. 300 Gemeindevertretern (Bürgermeister, Umweltgemeinderäte, Amtsleiter) beim 3. Interkommunalen Erfahrungsaustausch zur Abfallwirtschaft von Dr. Heinz-Josef Dornbusch - Geschäftsführer des Instituts für Abfall, Abwasser und Infrastruktur-Management $\mathrm{GmbH}$ (INFA) in Ahlen (D), welches mit der Durchführung des Projektes beauftragt war - präsentiert.

Mittlerweile liegen sehr viele positive Rückmeldungen der teilnehmenden Gemeinden vor bzw. haben viele Gemeinden angeregt, das Projekt im Jahr 2009 zu wiederholen, um damit auch an diesem Benchmarking teilnehmen zu können. Diese Anregung wurde von der FA19D gerne angenommen und die INFA GmbH wegen einer Fortführung des Projektes kontaktiert.

Auf der Website der FA19D können Interessierte unter www.abfallwirtschaft.steiermark.at/cms/ beitrag/10933476/46548 einen anonymisierten Bericht downloaden.

Nachstehend sind die wesentlichen Ergebnisse des Projektes für die Kapitel Restmüll und Altstoffsammelzentren kurz zusammengefasst:

\section{Projektbeschreibung}

Ziel des Projektes „Steirischer Abfallspiegel“" war es, die abfallwirtschaftlichen Strukturen von Gemeinden (Bezugsjahr 2007) mit dem steirischen Durchschnitt zu verglei- chen. Teilnahmeberechtigt waren alle 542 Gemeinden im Land Steiermark. Auf der Website der FA19D wurde dazu ein Fragebogen mit einem detaillierten Definitionsteil sowie integrierter Kennzahlenermittlung bereitgestellt. Die umfangreichen Plausibilitätsprüfungen und Auswertungen wurden von der INFA GmbH vorgenommen. Nach Fertigstellung wurde jeder Teilnehmergemeinde ein Bericht übermittelt.

Dieser Auswertungsband enthält einen allgemeinen Teil, in dem die allgemeingültigen, d.h. gemeindeübergreifenden Ergebnisse dargestellt und bewertet werden. Im gemeindespezifischen Berichtsteil folgen dann die gemeindespezifischen Ergebnisse im Vergleich zur Teilnehmergruppe und mit einer Einstufung der Gemeindeergebnisse über eine sogenannte Ampelschaltung (von grün $=$ deutlich besser als die Vergleichsgemeinden bis rot $=$ deutlich schlechter als die Vergleichsgemeinden).

Der Bericht umfasst folgende abfallwirtschaftliche Themenbereiche:

- Restmüll,

- Sperrmüll,

- Bioabfall,

- Altpapier,

- Altstoffsammelzentrum,

- fraktionsübergreifende Darstellung ausgewählter Kennzahlen,

- Kosten-, Einnahmen- und Gebührenübersicht.

\section{Allgemeine Auswertungen}

Am Pilotprojekt haben sich erfreulicherweise 67 steirische Gemeinden beteiligt, wobei die kleinste Gemeinde ca. 300 Einwohner und die größte ca. 10.000 Einwohner hat. In allen 
Gemeinden sind Drittunternehmen mit der Abfuhr der Abfälle und Wertstoffe beauftragt. In nahezu allen Gemeinden ist die Anlieferung an Altstoffsammelzentren (gemeindeeigenes ASZ oder Nutzung in einer Gemeindekooperation) bzw. stationäre Sammelstellen möglich.

Einleitend werden strukturelle Rahmenbedingungen aufgezeigt, je Bereich (Restmüll, Bioabfall etc.) werden die Ergebnisse (aller teilnehmenden Gemeinden) in einem 3D-Kreisdiagramm dargestellt. Die ermittelten Kennzahlen werden je Bereich in einer tabellarischen Aufstellung aufbereitet. In der Tabelle sind folgende Inhalte dargestellt:

- Kurzbezeichnung der Kennzahl (inkl. Einheit)

- Stichprobenanzahl der jeweils teilnehmenden Gemeinden

- Untergrenze (10\% der Kennzahlenwerte aller Gemeinden liegen unterhalb dieses Wertes)

- Median (der Median ist der Kennzahlenwert, der von $50 \%$ der Gemeinden über- bzw. von $50 \%$ unterschritten wird)

- Obergrenze $(90 \%$ der Kennzahlenwerte aller Gemeinden liegen unterhalb dieses Wertes)

\section{Restmüll (gemischter Siedlungsabfall)}

In Abbildung 1 ist die Verteilung der Behältergrößen in Kombination mit der Serviceart über alle Gemeinden aufgezeigt.

In der Gesamtbetrachtung zeigt sich, dass ca. $83 \%$ aller Gefäße im Teilservice (Bürger stellt den Behälter zur Leerung an den Straßenrand, inkl. Sack) bereitgestellt werden. Das 120-Liter-Gefäß im Teilservice ist hierbei der am häufigsten bereitgestellte Behältertyp.

Abbildung 2 stellt die angebotenen Abfuhrintervalle dar.

Den eindeutigen Schwerpunkt in den Teilnehmergemeinden bildet mit $63 \%$ das 4-wöchentliche Leerungsintervall. Zudem wird ersichtlich, dass $92 \%$ aller Gemein-

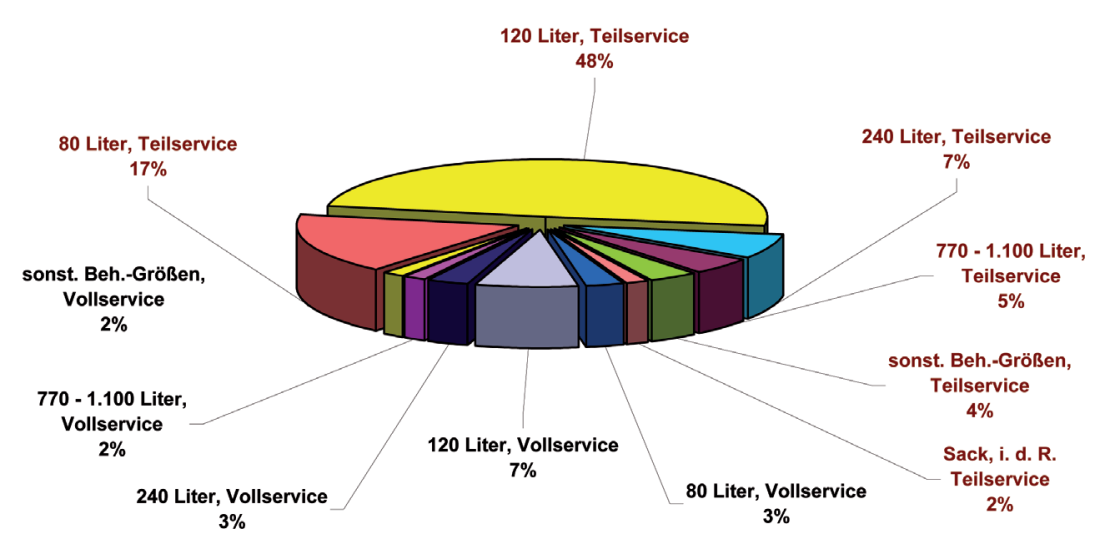

Abb. 1: Behälterverteilung Restmüll

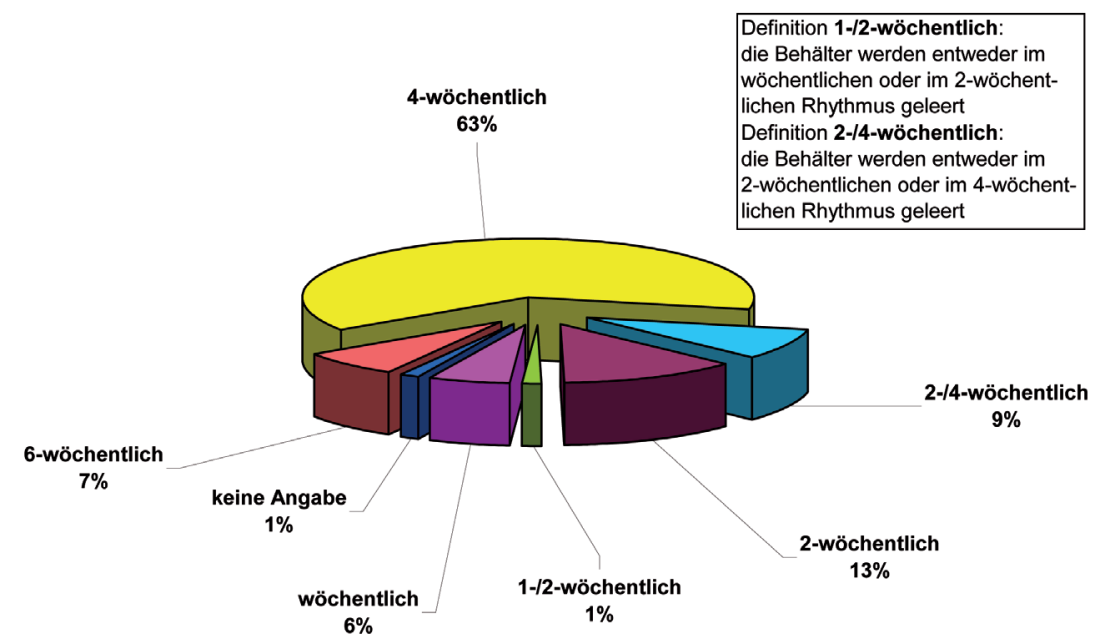

Abb. 2: Abfuhrintervalle Restmüll

\begin{tabular}{|c|c|c|c|c|c|}
\hline \multicolumn{2}{|c|}{$\begin{array}{l}\text { Restmüll } \\
\text { (gemischter Siedlungsabfall) }\end{array}$} & \multicolumn{4}{|c|}{ Vergleichswerte } \\
\hline Kennzahl & Einheit & $\begin{array}{l}\text { Anzahl } \\
\text { Ver- } \\
\text { gleichs- } \\
\text { werte }\end{array}$ & $\begin{array}{l}\text { Unter- } \\
\text { grenze }\end{array}$ & Median & $\begin{array}{l}\text { Ober- } \\
\text { grenze }\end{array}$ \\
\hline $\begin{array}{l}\text { bereitgestelltes Behälter } \\
\text { volumen }\end{array}$ & $\mathrm{I} /\left(E W^{*} \mathrm{a}\right)$ & 70 & 478 & 666 & 1.504 \\
\hline einwohnerspezifische Menge & $\mathrm{kg} /\left(E W^{*} \mathrm{a}\right)$ & 68 & 57,1 & 87,3 & 149,9 \\
\hline Sammelkosten pro Menge & $€ /$ Tonne & 64 & 76,1 & 131,3 & 193,4 \\
\hline Kosten Behandlung pro Menge & $€ /$ Tonne & 65 & 137,5 & 164,0 & 177,5 \\
\hline Gesamtkosten pro Menge & $€ /$ Tonne & 67 & 228,3 & 279,3 & 375,2 \\
\hline $\begin{array}{l}\text { Gesamtkosten pro Einwohner } \\
\text { und Jahr }\end{array}$ & $€ /\left(E W^{*} a\right)$ & 67 & 16,9 & 23,6 & 42,6 \\
\hline $\begin{array}{l}\text { Sammelkosten pro Behälter- } \\
\text { einheitenleerung }\end{array}$ & €/Be-Leer. & 65 & 1,6 & 2,4 & 4,2 \\
\hline
\end{tabular}

den ein Abfuhrintervall $\geq 2$ Wochen anbieten.

müll zusammen.

Pro abgefahrener Tonne Rest-

Tabelle 1 fasst die wesentlichen Kennzahlen aus dem Bereich Rest- müll fallen reine Sammelkosten

(Kosten für Personal, Fahrzeuge 


\begin{tabular}{|c|c|c|c|c|c|c|c|c|}
\hline \multirow[t]{2}{*}{ Abfuhrintervall } & \multicolumn{4}{|c|}{ Teilservice } & \multicolumn{4}{|c|}{ Vollservice } \\
\hline & $\begin{array}{l}\text { Beh.-Vol } \\
\text { I/(EW*a) }\end{array}$ & $\begin{array}{c}\text { spez. } \\
\text { Menge } \\
\text { kg/(EW*a) }\end{array}$ & $\begin{array}{c}\text { Sammel- } \\
\text { kosten je } \\
\text { Tonne } \\
\text { [€/Tonne] }\end{array}$ & $\begin{array}{c}\text { Gesamt- } \\
\text { kosten je } \\
\text { WE } \\
€ /\left(E W^{*} a\right)\end{array}$ & $\begin{array}{l}\text { Beh.-Vol } \\
\text { I/(EW*a) }\end{array}$ & $\begin{array}{c}\text { spez. } \\
\text { Menge } \\
\text { kg/(EW*a) }\end{array}$ & $\begin{array}{c}\text { Sammel- } \\
\text { kosten je } \\
\text { Tonne } \\
\text { [€/Tonne] }\end{array}$ & $\begin{array}{c}\text { Gesamt- } \\
\text { kosten je } \\
\text { EW } \\
€ /\left(E W^{\star} a\right)\end{array}$ \\
\hline 6-wöchentlich & 567 & 66,5 & 147,5 & 20,0 & \multicolumn{4}{|c|}{ keine Angaben } \\
\hline 4-wöchentlich & 584 & 81,5 & 126,8 & 23,4 & 796 & 106,5 & 153,4 & 36,2 \\
\hline 2-wöchentlich & 1.391 & 130,5 & 163,7 & 32,8 & 1.247 & 135,4 & 184,0 & 47,9 \\
\hline
\end{tabular}

und Behälter) in Höhe von im Mittel $131 €$ an. Die Behandlungskosten pro Tonne liegen bei den Teilnehmergemeinden in einem vergleichweise engen Wertebereich von ca. 138 bis $178 €$ pro Tonne. Die Gesamtkosten bilden die Summe aus den Sammelkosten und den Behandlungskosten und liegen beim Restmüll im Mittel bei $279 €$ pro Tonne. Bezogen auf den Einwohner lassen sich Kosten von im Mittel ca. $24 € /\left(\mathrm{EW}^{*} \mathrm{a}\right)$ erkennen, die Sammelkosten pro Behältereinheitenleerung liegen bei ca. 2,40€/Be-Leer.

Tabelle 2 zeigt die Ergebnisse einer vertiefenden Betrachtung hinsichtlich der Einflüsse „Abfuhrintervall“ und „Serviceart" auf die erfassten Restmüllmengen und die entstehenden Kosten.

Bei der Detailanalyse zeigt sich, dass das spezifische Behältervolumen mit zunehmender Leerungshäufigkeitsteigt; mitzunehmendem Behältervolumen steigen parallel auch die spezifischen Mengen pro Einwohner und Jahr. Unter Berücksichtigung dieser Faktoren steigen im Regelfall zudem die Sammelkosten pro Tonne (Ausnahme hier bei 4-wöchentlichem Teilservice) als auch die Gesamtkosten je Einwohner und Jahr. Die Differenzierung zwischen Voll- und Teilservice weist für den Vollservice jeweils höhere Sammel- und Gesamtkosten aus.

\section{Altstoffsammelzentrum}

Das Altstoffsammelzentrum (nachfolgend ASZ) bildet in einer Gemeinde oder einer Gemeindekoo-

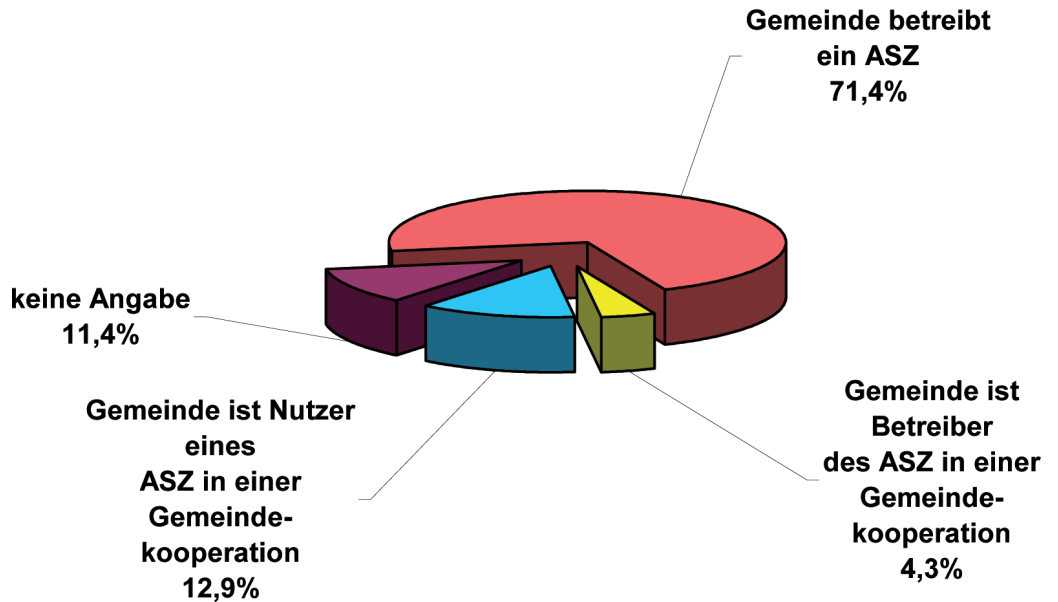

Abb. 3: Betrieb und Nutzung der Altstoffsammelzentren

Tab. 3: Kennzahlen Altstoffsammelzentren

\begin{tabular}{|c|c|c|c|c|c|}
\hline \multicolumn{2}{|l|}{$\begin{array}{l}\text { Kennzahlen zum } \\
\text { Altstoffsammelzentrum }\end{array}$} & \multicolumn{4}{|c|}{ Vergleichswerte } \\
\hline Kennzahl & Einheit & $\begin{array}{c}\text { Anzahl } \\
\text { Ver- } \\
\text { gleichs- } \\
\text { werte }\end{array}$ & $\begin{array}{l}\text { Unter- } \\
\text { grenze }\end{array}$ & Median & $\begin{array}{l}\text { Ober- } \\
\text { grenze }\end{array}$ \\
\hline Einwohner pro ASZ & EW/ASZ & 57 & 1.399 & 2.336 & 7.482 \\
\hline Öffnungszeiten pro Woche & h/wo & 54 & 0,8 & 2,0 & 11,0 \\
\hline $\begin{array}{l}\text { Mitarbeiterminuten } \\
\text { Betreuung pro Einwohner }\end{array}$ & $\min /\left(E W^{\star} a\right)$ & 53 & 4 & 10 & 31 \\
\hline $\begin{array}{l}\text { Anlieferungen pro } \\
\text { Öffnungsstunde }\end{array}$ & Anl./h & 46 & 4,2 & 14,8 & 30,8 \\
\hline $\begin{array}{l}\text { Durchsatzmenge pro } \\
\text { Öffnungsstunde }\end{array}$ & $\mathrm{kg} / \mathrm{h}$ & 54 & 392 & 2.302 & 8.679 \\
\hline $\begin{array}{l}\text { Durchsatzmenge pro } \\
\text { Einwohner im Einzugsgebiet }\end{array}$ & $\mathrm{kg} /\left(\mathrm{EW}^{*} \mathrm{a}\right)$ & 56 & 44,3 & 112,0 & 252,1 \\
\hline $\begin{array}{l}\text { Personalkosten pro } \\
\text { Öffnungsstunde }\end{array}$ & $€ / \mathrm{h}$ & 47 & 19,7 & 58,2 & 139,8 \\
\hline $\begin{array}{l}\text { Betriebskosten pro } \\
\text { Öffnungsstunde }\end{array}$ & $€ / \mathrm{h}$ & 48 & 6,3 & 19,5 & 127,1 \\
\hline Betriebskosten pro Einwohner & $€ /\left(E W^{*} a\right)$ & 49 & 0,2 & 1,9 & 7,4 \\
\hline Erlöse pro Einwohner & $€ /\left(E W^{*} a\right)$ & 52 & 0,6 & 2,1 & 6,7 \\
\hline $\begin{array}{l}\text { Gesamtkosten pro Einwohner } \\
\text { und Jahr (nach Abzug Erlöse) }\end{array}$ & $€ /\left(E W^{*} a\right)$ & 47 & 7,6 & 14,5 & 26,4 \\
\hline
\end{tabular}




\section{Tab. 4: Kennzahlen in fraktionsübergreifender Darstellung}

\begin{tabular}{|c|c|c|c|c|c|}
\hline \multicolumn{2}{|c|}{$\begin{array}{l}\text { Fraktionsübergreifende Darstellung } \\
\text { der einwohnerspezifischen Mengen } \\
\text { (ausgewählte Fraktionen) }\end{array}$} & \multicolumn{4}{|c|}{ Vergleichswerte } \\
\hline Kennzahl & Einheit & $\begin{array}{c}\text { Anzahl } \\
\text { Ver- } \\
\text { gleichs- } \\
\text { werte }\end{array}$ & $\begin{array}{l}\text { Unter- } \\
\text { grenze }\end{array}$ & Median & $\begin{array}{l}\text { Ober- } \\
\text { grenze }\end{array}$ \\
\hline $\begin{array}{l}\text { Gesamtmenge } \\
\text { (ausgew. Fraktionen) }{ }^{11} \\
\text { davon: }\end{array}$ & kg/(EW*a) & 68 & 154,0 & 258,6 & 415,2 \\
\hline Restmüll & $\mathrm{kg} /\left(E W^{*} \mathrm{a}\right)$ & 68 & 57,1 & 87,3 & 149,9 \\
\hline $\begin{array}{l}\text { Sperrmüll (mobile Samml. } \\
+ \text { ASZ) }\end{array}$ & $\mathrm{kg} /\left(\mathrm{EW}^{\star} \mathrm{a}\right)$ & 62 & 13,2 & 25,6 & 50,1 \\
\hline $\begin{array}{l}\text { Altholz (mobile Samml. } \\
+ \text { ASZ) }\end{array}$ & kg/(EW*a) & 54 & 3,5 & 13,6 & 31,3 \\
\hline $\begin{array}{l}\text { Bioabfall (inkl. biogene } \\
\text { Abfälle aus ASZ) }\end{array}$ & $\mathrm{kg} /\left(E W^{*} \mathrm{a}\right)$ & 57 & 5,6 & 50,9 & 159,2 \\
\hline Altpapier (inkl. ASZ) & $\mathrm{kg} /\left(\mathrm{EW}^{*} \mathrm{a}\right)$ & 67 & 55,5 & 72,8 & 98,2 \\
\hline Altmetalle (ASZ) & $\mathrm{kg} /\left(\mathrm{EW}^{*} \mathrm{a}\right)$ & 53 & 4,2 & 10,7 & 23,5 \\
\hline Wertstoffabschöpfung ${ }^{2)}$ & $\%$ & 68 & 39,4 & $56,1^{3)}$ & 66,9 \\
\hline
\end{tabular}

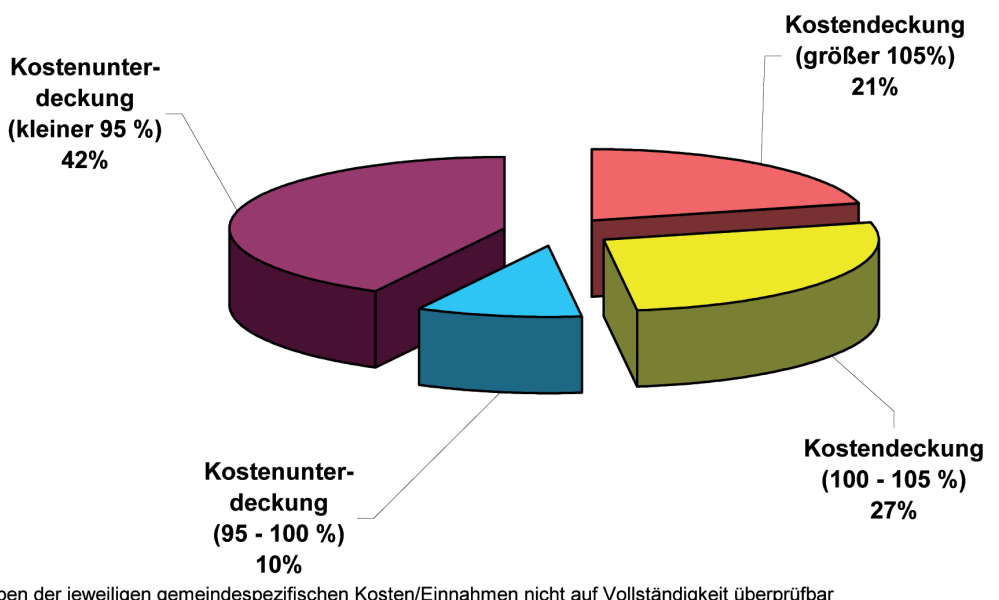

*) Angaben der jeweiligen gemeindespezifischen Kosten/Einnahmen nicht auf Vollständigkeit überprüfbar

Abb. 4: Übersicht über die Art der Kostendeckung

\begin{tabular}{|c|c|c|c|c|c|}
\hline \multicolumn{2}{|c|}{$\begin{array}{l}\text { Kosten-, Einnahmen- und } \\
\text { Gebührenübersicht }\end{array}$} & \multicolumn{4}{|c|}{ Vergleichswerte } \\
\hline Kennzahl & Einheit & $\begin{array}{c}\text { Anzahl } \\
\text { Ver- } \\
\text { gleichs- } \\
\text { werte }\end{array}$ & $\begin{array}{l}\text { Unter- } \\
\text { grenze }\end{array}$ & Median & $\begin{array}{l}\text { Ober- } \\
\text { grenze }\end{array}$ \\
\hline $\begin{array}{l}\text { Gesamtkosten Abfall- } \\
\text { wirtschaft }\end{array}$ & $E /\left(E W^{*} a\right)$ & 69 & 39,9 & 64,1 & 90,0 \\
\hline $\begin{array}{l}\text { Einnahmen, Erlöse und } \\
\text { Gebühreneinnahmen }\end{array}$ & $E /\left(E W^{*} a\right)$ & 69 & 35,1 & 57,7 & 98,4 \\
\hline
\end{tabular}

peration die zentrale Anlaufstelle zur Abgabe aller weiteren Fraktionen, die über die klassisch haushaltsnah erfassten Fraktionen Restmüll, Bioabfall und Altpapier hinaus anfallen.

Abbildung 3 veranschaulicht die Ausgangssituation bei den Altstoffsammelzentren.

Mit $71,4 \%$ der teilnehmenden 72 Gemeinden betreibt der überwiegende Teil der Gemeinden ein eigenes ASZ, weitere $4,3 \%$ betreiben ein ASZ in einer Gemeindekooperation und 12,9\% der Gemeinden sind Nutzer eines ASZ in einer Gemeindekooperation.

Tabelle 3 zeigt die Aufstellung der relevanten Kennzahlen zu den ASZ.

Im Mittel ist ein ASZ 2 Stunden pro Woche geöffnet, wobei das ASZ dabei i.d.R. nur an 1 oder 2 Tagen pro Monat (dann aber für mehrere Stunden) geöffnet hat. Der Betreuungsaufwand durch ASZ-Personal liegt im Mittel bei ca. 10 Minuten pro Einwohner und Jahr. Je Öffnungsstunde fallen im Mittel ca. 15 Anlieferungen an.

Je nach Intensität der Betreuung liegen die Personalkosten bei ca. $58 €$ pro Öffnungsstunde und die Betriebskosten von ASZ (ohne Personalkosten) im Mittel bei ca. $20 € / h$.

Die durch die Annahme bestimmter Fraktionen erzielten Erlöse liegen im Mittel bei ca. 2,1€ pro Einwohner und Jahr. Berücksichtigt man diese wiederum kostenmindernd bei der Ermittlung der Kosten für Personal, für den Betrieb und die Entsorgung der erfassten Mengen am ASZ (= Gesamtkosten), so fallen im Mittel Kosten von ca. $14,5 €$ pro Einwohner und Jahr an.

\section{Fraktionsübergreifende Darstellungen}

In Tabelle 4 sind in einer Gesamtübersicht die einwohnerspezifischen Mengen ausgewählter Fraktionen dargestellt. Bei diesem 
Ansatz werden unter anderem die Mengen aus dem Holsystem (Erfassung am Haushalt/haushaltsnahe Sammelstellen/mobile Sammlung) mit den z.T. zusätzlich am ASZ erfassten Mengen in Summe dargestellt (siehe Sperrmüll, Altholz, Bioabfall, Altpapier).

Bei der Aufsummierung der ausgewählten Fraktionen zeigt sich eine durchschnittliche Gesamtmenge je Einwohner und Jahr von ca. 259 Kilogramm. Erkennbar sind hier z.T. deutliche Schwankungen, die zwischen 154 bis 415 Kilogramm pro Einwohner und Jahr liegen. Die Wertstoffabschöpfung liegt im Mittel bei $56 \%$

\section{Kosten, Einnahmen und Gebühren}

Alle abfallwirtschaftlichen Kosten (inkl. Tierkörperbeseitigung, Standplatzreinigung etc.) wurden abschließend abgefragt und den Erlösen/Einnahmen (bei der Annahme bestimmter Fraktionen) sowie den Gebühreneinnahmen gegenübergestellt (Tabelle 5).

Auf dieser Basis lässt sich pro Gemeinde eine Kostendeckung oder Kostenunterdeckung ausweisen. Eine Verteilung über alle Teilnehmergemeinden ist in Abbildung 4 dargestellt.

Wie aus der Abbildung ersichtlich wird, erzielen ca. $48 \%$ der Teilnehmergemeinden eine Kostendeckung (größer/gleich 100\%) bei der Gegenüberstellung der erzielten Einnahmen/Erlöse/Gebühreneinnahmen und den abfallwirtschaftlichen Gesamtkosten, weitere $10 \%$ liegen in einem Bereich von $95-100 \%$ Kostendeckung und weisen somit nur eine leichte Unterdeckung auf.

\section{Zusammenfassung der Allgemeinen Auswertung}

Im Rahmen der Datenerhebungen zum Pilotprojekt „Steirischen Abfallspiegel“ konnte eine Vielzahl abfallwirtschaftlicher Kennzahlen für die 67 Teilnehmergemeinden ermittelt werden. Die dargestellten Ergebnisse liefern einen ersten Überblick über die allgemeine abfallwirtschaftliche Situation in der Steiermark und dienen im Wesentlichen einer ersten Standortbestimmung.

Mögen die aus dieser Arbeit zu

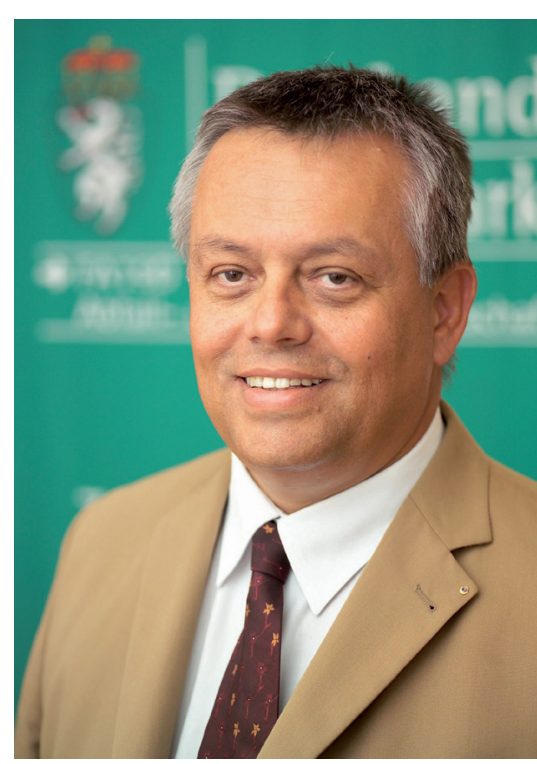

HR DI Dr. Wilhelm Himmel

ziehenden Erkenntnisse einen Beitrag zur Optimierung abfallwirtschaftlicher Prozesse in den Gemeinden leisten!

Informationen:

Amt der Steiermärkischen Landesregierung

FA19D - Abfall- und Stoffflusswirtschaft

Abt.-Leiter Hofrat DI Dr. Wilhelm Himmel

Bürgergasse $5 \mathrm{a}$

8010 Graz

Tel. +43-316-877-4323, Fax +43-316-877-2416

fa19d@stmk.gv.at

www.abfallwirtschaft.steiermark.at 